\title{
Efficient optical pumping of organic-inorganic heterostructures for nonlinear optics
}

\author{
V. M. Agranovich, ${ }^{1,2}$ D. M. Basko, ${ }^{3}$ and G. C. La Rocca ${ }^{4}$ \\ ${ }^{1}$ NanoTech Institute, Chemistry Department, The University of Texas at Dallas, Richardson, Texas 75083, United States \\ ${ }^{2}$ Institute of Spectroscopy, Russian Academy of Science, Troitsk, Moscow Region 142190, Russia \\ ${ }^{3}$ Université Grenoble 1/CNRS, LPMMC UMR 5493, 25 rue des Martyrs, 38042 Grenoble, France \\ ${ }^{4}$ Scuola Normale Superiore and CNISM, Piazza dei Cavalieri, 56126 Pisa, Italy
}

\begin{abstract}
We theoretically consider an hybrid heterostructure made of an inorganic quantum well in close proximity with an organic material overlayer whereby the latter is used to funnel excitation energy to the former in order to exploit the optical nonlinearities of the two-dimensional Wannier excitons. On the one hand, the diffusion length of Frenkel excitons in the organic medium is assumed to be comparable or larger than the corresponding absorption length. On the other hand, the nonradiative energy transfer from the organic to the inorganic subsytem can be very efficient when the Frenkel exciton energy is significantly higher than the band gap of the inorganic semiconductor. We show in this regime that the resonant optical pumping of the Frenkel excitons can lead to an efficient indirect pumping of the Wannier excitons (or electron-hole plasma) in the inorganic quantum well turning on the corresponding nonlinearities.
\end{abstract}

PACS numbers: 78.66.-w ; 78.20.Bh ; 78.66.Qn

\section{INTRODUCTION}

The electronic states and the optical properties of hybrid organic-inorganic nanostructures have attracted much interest both theoretically and experimentally (we refer the reader to the recent review Ref. 1, and references therein). It was predicted, in particular, that a substantial enhancement of the resonant optical $\chi^{(3)}$ nonlinearity can be achieved in strongly coupled hybrid structures in which the eigenstates are coherent superposition of Wannier and Frenkel exciton states of the inorganic quantum well (QW) and the organic overlayer subsystem, respectively ${ }^{2}$. To the best of our knowledge, however, this regime of enhanced nonlinearity has not yet been demonstrated experimentally. We focus here on the issue of the optical nonlinearity in hybrid structures in the weak coupling regime in which no hybrid excitons are formed, a regime which is more easily accessible experimentally. The optical nonlinearity of the inorganic QW is proportional to the concentration of excitons and e-h pairs in the well ${ }^{3}$. Nonlinear effects on the QW exciton resonance are observed also at room temperature and are large enough to be used for device applications ${ }^{4}$. We will argue below that an enhancement of the nonlinearity of the semiconductor QW could take place in the weak coupling regime as well and these systems may also provide a possibility to vary the $\mathrm{QW}$ nonlinearity optically by changing the pumping intensity of the organic overlayer.

The resonant absorption of light by a thin QW and the direct creation of excitons and e-h pairs in the well is small. However, the excitation density (and subsequent nonlinearity) in a hybrid structure such as the one sketched in Fig.1 can be increased due to the strong absorption of incident light by the organic overlayer (having large oscillator strength) followed by non-radiative energy transfer to the inorganic QW. Such indirect pumping of the QW can be very effective if the light from the external pumping beam will resonantly generate Frenkel excitons in the organic layer with energies significantly larger than the exciton energy in the semiconductor quantum well, i.e. at an energy at which the semiconductor already has a large absorption. The overall light absorption in the organic component of the hybrid structure can be much larger than the direct absorption by a thin QW so that the organic component plays in this case the role of a funnel of pumping radiation energy. This scheme can be viewed as complementary to that of pumping an organic medium via energy transfer from an inorganic semiconductor, proposed earlier ${ }^{5}$.

The process considered here can be effective if the absorption length of the organic material, i.e. the thickness required to absorb light in the organic subsystem, is not larger than the diffusion length of the Frenkel excitons, so that most of them will be able to get close to the organic-inorganic interface and to transfer their energy to the inorganic QW by the Förster mechanism, a process which is known to be quite efficient ${ }^{1}$. Following this non-radiative energy transfer and subsequent energy relaxation, the QW in the hybrid structure can reach a state with a high excitation density and thus exhibit a number of nonlinear optical effects which have attracted much attention ${ }^{4}$. The QW Wannier exciton line which can be observed even at room temperature shifts ${ }^{6}$ and its oscillator strength is suppressed ${ }^{7}$ while its linewidth broadens $^{8}$ with increasing pumping until the exciton saturation density is reached and the Mott transition to an electron-hole plasma takes place ${ }^{9}$. Such optical nonlinearities would be relevant for a probe beam of light resonant with the QW exciton transition. As this probe beam has a frequency appreciably lower than the Frenkel exciton frequency in the organic overlayer, it would not be absorbed in the organic part of the hybrid structure. Variations in the pumping intensity of the organic component would produce, via non-radiative energy transfer to the inorganic quantum well, variations in the concentration of Wannier excitons in the QW, and thus optical 


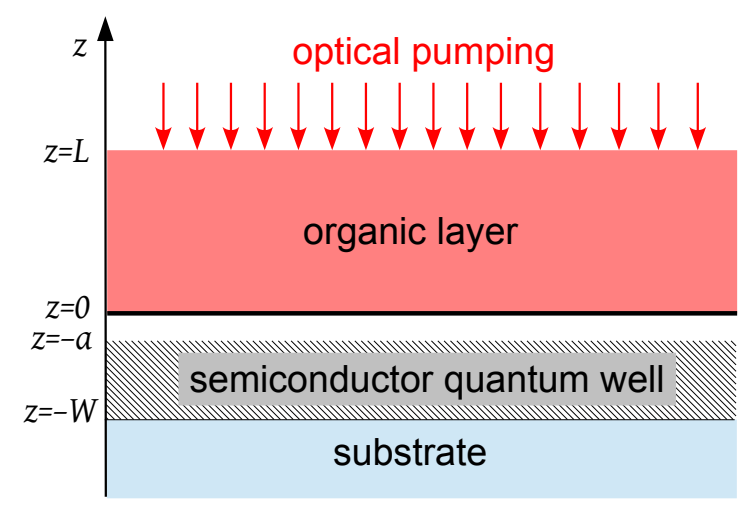

FIG. 1. (color online) Sketch of the hybrid heterostructure. In the organic overlayer (having thickness $L$ and absorption coefficient $\alpha$ ) Frenkel excitons are generated by optical pumping and diffuse (with diffusion length $L_{D}$ ) to the organic-inorganic interface, whereupon they non-radiatively transfer (with effective Förster radius $R_{0}$ ) to the inorganic semiconductor subsystem of thickness $W+a$ comprising a quantum well and its barriers ( $a \ll W$ being the thickness of an interfacial microscopic dead layer). The indirect pumping of the quantum well is efficient provided $a \ll R_{0} \sim W \ll L \sim \alpha^{-1}<L_{D}$ (see text for details).

control of the QW nonlinearity can be achieved.

For a thin QW in close proximity with the organic overlayer, this mechanism of indirect nonresonant pumping turns out to be more efficient than the one via direct optical absorption in the inorganic medium. We calculate here in detail the efficiency of such indirect optical pumping assuming for illustrative purposes values of material parameters appropriate for a hybrid heterostructure based on II-VI inorganic semiconductors and on anthracene at room temperature. In particular, we also provide an analytical expression for the efficiency [see Eqs. (4), (5) below] which is very accurate for most cases of practical interest and could be used for other materials as well. While these results appear to be fairly simple, their validity hinges on a rather technical scrutiny accounting for the specific dependence on distance of the energy transfer. For the sake of completeness, the Appendix reports the details of such analysis showing, in particular, how in the cases of interest here the effects of the energy transfer can be described in terms of an effective boundary condition for the steady state diffusion equation.

\section{THE MODEL}

The model hybrid heterostructure we study is shown in Fig.1. The highest efficiency of the process of indirect optical pumping we consider here requires that the inorganic semiconductor material be strongly absorbing at the energy of the Frenkel exciton in the organics, and that the Frenkel exciton diffusion length $L_{D}$ and the absorption coefficient $\alpha$ of the organic material be such that $\alpha L_{D} \gtrsim 1$. The Frenkel excitons are described as point dipole excitations (incoherent molecular excitons) in the diffusive regime, i.e. with a hopping time much shorter than their lifetime $\tau$. For illustrative purposes, we make use of material parameters typical of anthracene $e^{10-14}$ for the organic overlayer and of a thin QW of II-VI semiconductors for the inorganic subsystem ${ }^{15,16}$. The absorption coefficient of anthracene at the Frenkel exciton energy of about $3.1 \mathrm{eV}$ is $\alpha \sim 3.2 \cdot 10^{5} \mathrm{~cm}^{-1}$ and the Frenkel exciton diffusion coefficient is $D=L_{D}^{2} / \tau \sim 5 \cdot 10^{-3} \mathrm{~cm}^{2} / \mathrm{s}$, being $\tau \sim 5 \mathrm{~ns}$ and the diffusion length $L_{D} \sim 500 \AA$. The inorganic subsystem should have a band gap significantly smaller than the Frenkel exciton energy and in the calculation of the non-radiative energy transfer rate (i.e. of the effective Förster radius $R_{0}$ ), it will be considered as a homogeneous medium described by a complex dielectric function. For a $\mathrm{Zn}(\mathrm{Cd})$ Se based quantum well (the $\mathrm{ZnSe}$ band gap is about $2.6 \mathrm{eV}$ ) at the Frenkel exciton energy the dielectric function is $\tilde{\varepsilon} \equiv(n+i \kappa)^{2} \approx(3+i)^{2}$.

Under continuous pumping, the diffusion equation for the Frenkel exciton density $\rho$ in the organic overlayer reduces to

$$
\alpha I_{0} e^{\alpha(z-L)}-\frac{\rho}{\tau}+\frac{L_{D}^{2}}{\tau} \frac{\partial^{2} \rho}{\partial z^{2}}-\Gamma(z) \rho=\frac{\partial \rho}{\partial t}=0,
$$

supplemented by the zero-current boundary conditions,

$$
\left.\frac{\partial \rho}{\partial z}\right|_{z=0}=\left.\frac{\partial \rho}{\partial z}\right|_{z=L}=0
$$

The first term in Eq. (1) represents exciton generation by the light absorption, $I_{0}$ being the photon flux which penetrates the organic layer. The second term accounts for the decay of excitons with the lifetime $\tau$, as due to spontaneous emission, or other quenching channels such as internal conversion or trapping by an impurity. The third term describes the diffusive transport. Finally, the last term in Eq. (1) represents the Förster transfer of excitons from the organic layer to the inorganic semiconductor quantum well with a rate $\Gamma(z)$ strongly decreasing with the distance $z$ from the interface.

The calculation of the transfer rate can be performed following the approach of Ref. 17. First, the electric field, which is due to the oscillating polarization of the Frenkel exciton and penetrates into the inorganic subsystem, is calculated neglecting retardation. Then, the rate of nonradiative energy transfer is obtained from the Joule losses proportional to the imaginary part of the dielectric response of the inorganic medium at the corresponding frequency. Taking into account both the finite well width and the dielectric mismatch, the result is

$$
\begin{aligned}
\Gamma(z)= & \frac{2 \operatorname{Im} \tilde{\varepsilon}}{\hbar} \int_{0}^{\infty} d k k^{2} e^{-2 k(z+a)}\left(d_{\|}^{2} / 2+d_{z}^{2}\right) \sinh k W \\
& \times \frac{\left|\tilde{\varepsilon}+\varepsilon_{s}\right|^{2} e^{k W}+\left|\tilde{\varepsilon}-\varepsilon_{s}\right|^{2} e^{-k W}}{\left|\tilde{\varepsilon}\left(\varepsilon+\varepsilon_{s}\right) \cosh k W+\left(\tilde{\varepsilon}^{2}+\varepsilon \varepsilon_{s}\right) \sinh k W\right|^{2}} .
\end{aligned}
$$


Here, $d_{\|}$and $d_{z}$ are the in-plane and perpendicular components of the transition dipole of the Frenkel exciton; $\varepsilon$, $\tilde{\varepsilon}$ and $\varepsilon_{s}$ are the dielectric functions of, respectively, the organic material, the inorganic semiconductor, and the transparent substrate. For the purpose of calculating the dielectric mismatch, the dielectric function of the organic material has been here considered isotropic. The effects of anisotropy on the energy transfer process can also be calculated similarly, but do not affect much the energy transfer efficiency ${ }^{18}$. Finally, the length $a$ represents a short-range cutoff ( $a \sim 10 \AA)$, corresponding physically to a microscopic distance being of the order of the lattice constant if the organics is deposited directly on the semiconductor, or, less optimistically, to the thickness of an exciton dead layer at the semiconductor boundary. The latter may also partly account for the presence of inhomogeneities at the organic-inorganic interface the suppression of which hinges on challenging technological issues $^{19,20}$.

The result for the transfer rate given above differs from those previously obtained ${ }^{1,17}$ mainly because the polarization source is here essentially point-like (due to incoherent molecular excitons) and in Eq. (3a) the integral is extended over all values of the two-dimensional wavevector, whereas in previous works the polarization source associated to Wannier excitons requires an integration over a thermal distribution of wavevectors or, for localized excitons, over a range limited by the inverse of the localization length. Yet, we are unable to express the integral in Eq. (3a) in terms of elementary functions, and we will not use in the following this general expression as is. It turns out that, for most cases of practical interest (namely, $W \gg a$, and $|\varepsilon|,|\tilde{\varepsilon}| \gg \varepsilon_{s}$ ), the function $\Gamma(z)$ from Eq. (3a) can be well approximated by a much simpler expression,

$$
\Gamma(z) \simeq \frac{\operatorname{Im} \tilde{\varepsilon}}{\hbar|\varepsilon+\tilde{\varepsilon}|^{2}} \frac{d_{\|}^{2} / 2+d_{z}^{2}}{(z+a)^{3}} \equiv \frac{1}{\tau} \frac{R_{0}^{3}}{(z+a)^{3}} .
$$

We note that the $z$ dependence in this expression can be obtained by a straightforward integration of the usual Förster rate $1 / r^{6}$ over the half-space $z<-a$. It is convenient to parametrize the strength of the Förster transfer by a length $R_{0}$, defined as the effective Förster radius by Eq. (3b). $R_{0}$ can be viewed as analogous to the usual Förster radius, adapted for our particular situation. Its value can be taken to match the full expression, Eq. (3a), at $z=0$. In the following, we stick to the simple model expression of Eq. (3b), which accurately reproduces all the relevant physics of Eq. (3a) in the cases of our interest.

It should be noted that $\operatorname{Im} \tilde{\varepsilon}$ enters Eqs. (3a), (3b) both in the numerator (since the transfer rate is proportional to the absorption in the semiconductor) and in the denominator (since the electric field penetrating the quantum well is screened by its dielectric constant). As in the denominator it enters squared, too strong absorption in the quantum well actually suppresses the transfer. In fact, the most advantageous situation is $\tilde{\varepsilon} \sim \varepsilon$. In this case, assuming that the short-distance cutoff $a$ is of the order of the lattice constant of the organic material, and taking into account the fact that the diffusion coefficient in many organic materials at room temperature is itself determined by the Förster transfer between the organic molecules ${ }^{21}$ one can obtain a simple estimate of the effective Förster radius $R_{0}$. Indeed, the diffusion coefficient can be estimated as

$$
D \sim \int_{|\mathbf{r}|>a} d^{3} \mathbf{r} r^{2} \frac{\operatorname{Im} \varepsilon}{\hbar|\varepsilon|^{2}} \frac{d^{2}}{r^{6}} \sim \frac{\operatorname{Im} \varepsilon}{\hbar|\varepsilon|^{2}} \frac{d^{2}}{a} \sim \frac{R_{0}^{3}}{\tau a}
$$

where in the last relation we have used Eq. (3b). This gives $R_{0}^{3} \sim L_{D}^{2} a$. This should be considered the most optimistic estimate of $R_{0}$, since increasing the dielectric mismatch, decreasing the absorption $\operatorname{Im} \tilde{\varepsilon}$, or increasing $a$ can only slow down the transfer. In anthracene $^{13}$ at the Frenkel exciton frequency $(3.12 \mathrm{eV})$ the dielectric constant along the strongly absorbing axis is $\varepsilon \equiv(n+i \kappa)^{2} \simeq(2.4+1.0 i)^{2}$, while in II-VI semiconductors ${ }^{16}$ such as $\mathrm{Zn}(\mathrm{Cd}) \mathrm{Se}$ at comparable frequencies $\tilde{\varepsilon} \equiv(n+i \kappa)^{2} \approx(3+i)^{2}$. Thus, the estimate $R_{0}^{3} \sim L_{D}^{2} a$ seems to be reasonable.

To give an idea of the precision of Eq. (3b), we mention that for $\varepsilon=\tilde{\varepsilon}=(3+i)^{2}, \varepsilon_{s}=3.75, a=10 \AA$, and $W=100 \AA$, Eq. (3b) with $R_{0}$, adjusted to reproduce $\Gamma(z=0)$, gives less than $10 \%$ error for $z<100 \AA$, and the precision increases when increasing the dielectric contrast (i.e., $\varepsilon \neq \tilde{\varepsilon}$ ). Taking $d_{\|}=3 \mathrm{D}, d_{z}=0$ (the anthracene exciton transition dipole ${ }^{12}$ ), we obtain $R_{0} \approx 70 \AA$, while $\left(L_{D}^{2} a\right)^{1 / 3} \approx 140 \AA$ [for numerical estimates it is convenient to keep in mind that $\left.(1 \mathrm{D})^{2} /(1 \AA)^{3} \approx 0.95 \hbar /(1 \mathrm{fs})\right]$. As expected, also in our configuration such value of the efficient Förster radius indicates that the non-radiative transfer process is not effective beyond a distance $100 \AA$, where the intrinsic relaxation in the organics takes over. Thus, we can conclude that $L \gg R_{0}$, so the dielectric mismatch at the front surface at $z=L$, ignored in Eq. (3a), does not affect the energy transfer rate. Most importantly, as discussed below, we will also be able to provide an analitycal solution of Eq. (1) based on the fact that $L_{D} \gg R_{0}$. Finally, we note the nearestneighbor hopping rate due to the dipole-dipole interaction is $\sim(1 / \hbar)\left(\operatorname{Im} \varepsilon /|\varepsilon|^{2}\right)\left(d^{2} / a^{3}\right) \sim 1 \mathrm{ps}^{-1}$.

\section{TRANSFER EFFICIENCY}

The transfer efficiency $\mathcal{E}$ (the ratio between the transferred population and the total number of incident photons $I_{0}$ ) can be straightforwardly calculated from the stationary solution of the diffusion equation (see Appendix 


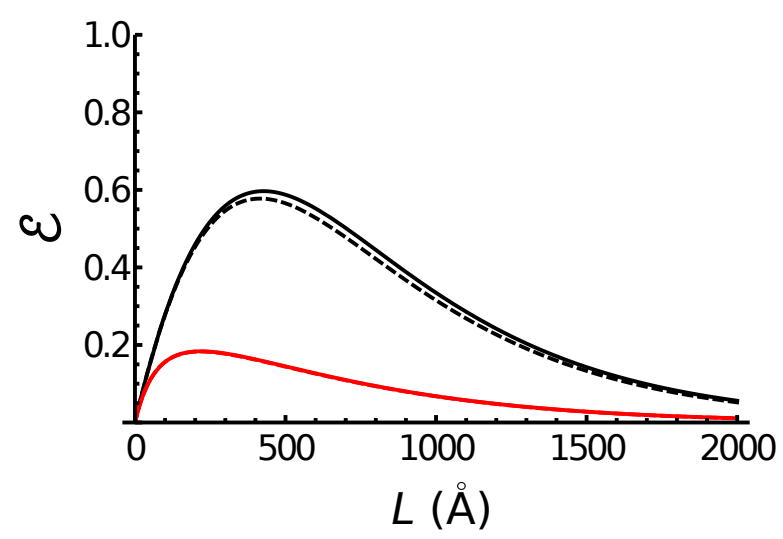

FIG. 2. (color online) Transfer efficiency $\mathcal{E}$ as a function of the organic layer thickness $L$ from the numerical solution of Eqs. (1), (3b) (solid lines) and from Eqs. (4), (5) (dashed lines, barely distinguishable from the solid ones) for two values of $R_{0}^{3} /\left(L_{D}^{2} a\right)=1,0.01$ (upper and lower curves, respectively), corresponding to $z_{0}$ about $20 \AA$ and $2000 \AA$. Other parameters are $\alpha^{-1}=300 \AA, L_{D}=500 \AA, a=10 \AA$.

for details):

$$
\begin{aligned}
\mathcal{E}= & \frac{\alpha^{2} L_{D}^{2}}{\alpha^{2} L_{D}^{2}-1} \times \\
& \times \frac{1-e^{-\alpha L}\left[\cosh \left(L / L_{D}\right)+\left(\alpha L_{D}\right)^{-1} \sinh \left(L / L_{D}\right)\right]}{\cosh \left(L / L_{D}\right)+\left(z_{0} / L_{D}\right) \sinh \left(L / L_{D}\right)},
\end{aligned}
$$

where the constant $z_{0}$ is given by

$$
\frac{1}{z_{0}}=\int_{0}^{\infty} \frac{\tau \Gamma(z)}{L_{D}^{2}} d z=\frac{R_{0}^{3}}{2 L_{D}^{2} a^{2}}
$$

One can distinguish two limiting cases. First, $\left|z_{0}\right| \ll$ $L_{D}$ corresponds to very efficient transfer, so the limiting factor for pumping the quantum well is the transport through the organic layer. In the second case, $z_{0} \gg L_{D}$, only a small fraction of excitons created in the organics will ever notice the presence of the semiconductor.

To illustrate our results, we plot in Fig. 2 the transfer efficiency obtained from Eqs. (4), (5), together with those obtained by numerical solution of Eq. (1) with $\Gamma(z)$ given by Eq. (3b). We use the material parameters corresponding to anthracene: $L_{D}=500 \AA, \alpha^{-1} \equiv(2 \kappa \omega / c)^{-1} \approx$ $300 \AA$ for light wavelength $400 \mathrm{~nm}$ and $\kappa \equiv \operatorname{Im} \sqrt{\varepsilon} \approx 1$, $a=10 \AA$, and the width of the quantum well $W=100 \AA$. As a function of the organic layer thickness $L$, the efficiency has a maximum at some optimal length $L_{o p t}$, whose physical meaning is quite transparent: when $L$ is too small (especially, when $L \ll \alpha^{-1}$ ), very few photons are absorbed, while when $L$ is too large (especially, when $L \gg L_{D}$ ), many excitons are lost in the organics and do not reach the organic-inorganic interface.

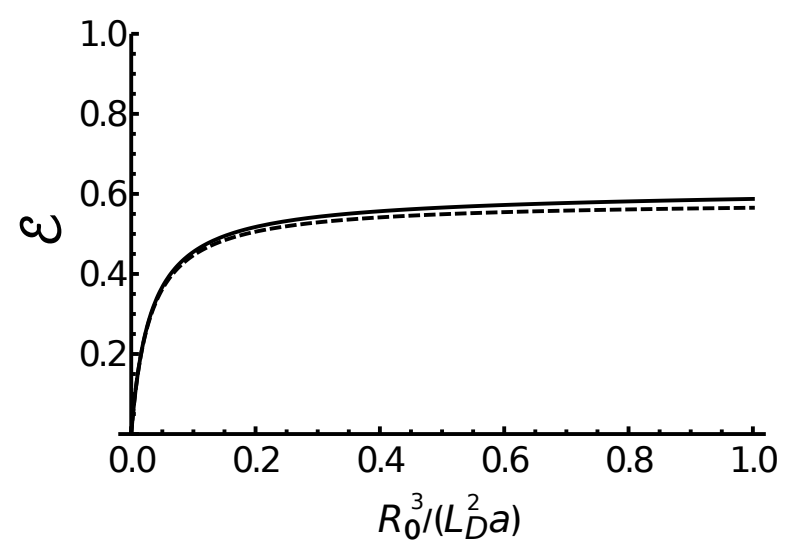

FIG. 3. Transfer efficiency $\mathcal{E}$ at $L=L_{D}$ as a function of $R_{0}^{3} /\left(L_{D}^{2} a\right)$ from the numerical solution of Eqs. (1), (3b) (solid line) and from Eqs. (4), (5) (dashed line). The rest of the parameters are as in Fig. 2.

Let us focus on the value of the efficiency at the maximum, since the layer thickness usually can be chosen appropriately. In Fig. 3 we plot the efficiency at $L=L_{D}$ (which approximately corresponds to the maximum) as a function of the dimensionless parameter $R_{0}^{3} /\left(L_{D}^{2} a\right)$, governing the Förster transfer strength. The maximum efficiency is reached at quite small values of $R_{0}^{3} /\left(L_{D}^{2} a\right)$. This can be easily understood from Eqs. (4), (5): the efficiency loses the sensitivity to the transfer rate when $z_{0} \ll L_{D}$, which occurs when $R_{0}^{3} /\left(L_{D}^{2} a\right) \gg 2 a / L_{D}$.

We also plot the maximum value of the efficiency, as obtained from Eq. (4) with $z_{0}=0$, and the corresponding optimal length as functions of the only remaining dimensionless parameter of the problem, $\alpha L_{D}$, see Fig. 4 . The efficiency can be arbitrarily close to unity for large $\alpha L_{D}$, and becomes small for $\alpha L_{D} \lesssim 1$, as in this case there is no way to satisfy the conditions $\alpha^{-1}<L<L_{D}$, which are necessary to combine efficient generation of excitons and efficient transport to the interface.

Finally, let us compare the obtained transfer efficiency $\mathcal{E}$ with the efficiency $\tilde{\mathcal{E}}$ of the direct optical pumping of the semiconductor quantum well in the absence of the organics. The latter is simply given by

$$
\tilde{\mathcal{E}}=1-e^{-\tilde{\alpha} W}
$$

where $\tilde{\alpha}=2(\omega / c) \tilde{\kappa}, \tilde{\kappa}=\operatorname{Im} \sqrt{\tilde{\varepsilon}}$. Taking again $\tilde{\alpha}^{-1}=$ $300 \AA$, for $W=100 \AA$ we obtain $\tilde{\mathcal{E}} \approx 0.3$, which is twice smaller than the maximal efficiency of the transfer via the organic layer, as calculated above. For a thinner quantum well, $\tilde{\mathcal{E}}$ will be even smaller, while $\mathcal{E}$ changes very little as long as $W>a$. The essential reason for this is the short-range nature of the Förster transfer as compared to the optical absorption length in the semiconductor. As a matter of fact, if a very thin semiconductor subsystem $(W \lesssim 50 \AA)$ could be employed on a transparent substrate, shining the pumping light from below through 


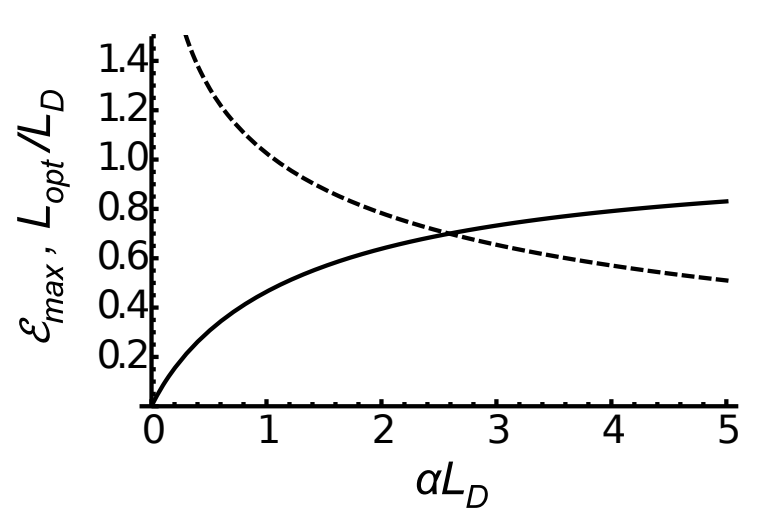

FIG. 4. The maximal transfer efficiency $\mathcal{E}_{\max }$ (solid line) and the optimal organic layer thickness $L_{\text {opt }}$ (in the units of $L_{D}$ ) at which this efficiency is reached (dashed line) as functions of $\alpha L_{D}$ from Eq. (4) with $z_{0}=0$.

the substrate (see Fig.1) could lead to an even higher efficiency of indirect pumping (which may depend on the dielectric contrast of the $\mathrm{QW} /$ substrate interface).

\section{CONCLUDING REMARKS}

We have considered a hybrid organic-inorganic heterostructure in which the organic subsystem is funneling the excitation energy which has optically absorbed into the inorganic quantum well. We have developed an analytical approach to calculate the efficiency of such indirect pumping which is very accurate for most cases of practical interest, and can be easily used for any combination of materials. The relevant physical scales governing the process have been identified, the crucial role being played by the adimensional parameters $\alpha L_{D}$ and $R_{0}^{3} /\left(L_{D}^{2} a\right)$. For large values of $\alpha L_{D} \gtrsim 1$ and $R_{0}^{3} /\left(L_{D}^{2} a\right) \sim 1$, the efficiency approaches $100 \%$, while for the typical illustrative cases here considered (based on material parameters appropriate for II-VI semiconductors and anthracene) it is of the order of $60 \%$. Finally, we remark that from the experimental point of view the realization of such a hybrid organic-inorganic heterostructure working in the weak coupling regime (i.e., not requiring the formation of coherent hybrid Wannier-Frenkel excitons) should be within the reach of state of the art techniques.

In a typical non degenerate pump and probe configuration (the energy of the pump being significantly higher than that of the Wannier exciton which is resonantly probed), this process can efficiently turn on the QW exciton nonlinearities in the inorganic subsystem, better than its direct optical pumping and in a spatially selective way in the sense that it produces a much stronger spatial concentration of excitations in the inorganic subsystem close to the organic-inorganic interface. These nonlinearities are proportional (at moderate pumping) to the exciton population incoherently generated in the QW following the non-radiative energy transfer, and have a recovery time limited by the Frenkel exciton lifetime in the organics (typically several nanoseconds). The indirect pumping here proposed could also be employed in a microcavity configuration ${ }^{22}$ in which the QW excitons are strongly coupled to the cavity photons and cavity polaritons are formed ${ }^{23}$. In such systems much attention has recently been devoted to many-body effects including the formation of a polariton condensate and superfluidity $^{24}$.

\section{ACKNOWLEDGEMENTS}

This work is supported by the European FP7 ICARUS program, grant agreement no. 237900. V. M. A. and D. M. B. thank Scuola Normale Superiore, where this work was done. V. M. A. also thanks the Russian Foundation of Basic Research for partial support.

\section{Appendix A: Solution of the diffusion equation and effective boundary conditions}

As the transfer occurs in the vicinity of the organicinorganic interface $z=0$, the full diffusion equation with the bulk Förster term, Eq. (1), sufficiently far from the interface can be replaced by the same equation without the last term,

$$
\alpha I_{0} e^{\alpha(z-L)}-\frac{\rho}{\tau}+\frac{L_{D}^{2}}{\tau} \frac{\partial^{2} \rho}{\partial z^{2}}=\frac{\partial \rho}{\partial t}=0,
$$

but with a modified boundary condition at the interface,

$$
\left.\left(\rho-z_{0} \frac{\partial \rho}{\partial z}\right)\right|_{z=0}=0 .
$$

The length $z_{0}$ should then be determined as follows. On the one hand, at small $z \ll L, L_{D}$, the solution of Eq. (A1) can be approximated by a linear function,

$$
\rho(z) \approx \rho(z=0)+\left.\frac{\partial \rho}{\partial z}\right|_{z=0} z .
$$

On the other, at such small $z$ we can neglect all terms in the original Eq. (1), except the last two, which becomes

$$
\frac{L_{D}^{2}}{\tau} \frac{\partial^{2} \rho}{\partial z^{2}}=\Gamma(z) \rho,
$$

considered in the half-space $z>0$, and supplemented by the zero-current boundary condition at $z=0$, see Eq. (2). At large $z \gg a$, the solution of Eq. (A4) is expected to have the form

$$
\rho(z \rightarrow \infty)=A+B z,
$$

where the coefficients $A$ and $B$ are not arbitrary, but have a fixed ratio (since the boundary condition at $z=0$ 
is fixed). Since the region of validity of Eq. (A3), $z \ll$ $L, L_{D}$, and that of Eq. (A5), $z \gg a$, overlap, one can match the solutions at $a \ll z \ll L, L_{D}$. This gives $z_{0}=$ $A / B$, which thus should be found from the solution of Eq. (A4).

Before finding $z_{0}$, we give the explicit form of the stationary solution of Eq. (A1) with the boundary condition (A2) at $z=0$ and zero current at $z=L$ :

$$
\begin{aligned}
& \rho(z)=\frac{\alpha I_{0} \tau}{\alpha^{2} L_{D}^{2}-1} \times \\
& \times\left[\alpha L_{D} \sinh \frac{L-z}{L_{D}}+\mathcal{A} \cosh \frac{z-z_{0}}{L_{D}}-e^{\alpha(z-L)}\right], \\
& \mathcal{A} \equiv \frac{\alpha L_{D} \mathcal{S}+\left(z_{0} / L_{D}\right) \mathcal{C}+\left(1-\alpha z_{0}\right) e^{-\alpha L}}{\mathcal{C}+\left(z_{0} / L_{D}\right) \mathcal{S}} \\
& \mathcal{C} \equiv \cosh \frac{L}{L_{D}}, \quad \mathcal{S} \equiv \sinh \frac{L}{L_{D}}
\end{aligned}
$$

The exciton population, transferred to the inorganic semiconductor quantum well per unit time, which for the full Eq. (1) would be given by $\int \Gamma(z) \rho(z) d z$, is now simply determined by the current at the interface:

$$
\mathcal{E}=\left.\frac{L_{D}^{2}}{I_{0} \tau} \frac{\partial \rho}{\partial z}\right|_{z=0},
$$

which gives Eq. (4).

Thus, all necessary information about the transfer is encoded in the constant $z_{0}$, or, more precisely, in the ratio $z_{0} / L_{D}$. One can distinguish two limiting cases. First, when $\left|z_{0}\right| \ll L_{D}$, the boundary condition is effectively $\rho(z=0)=0$. This corresponds to very efficient transfer, so the limiting factor for pumping the quantum well is the transport through the organic layer. Second, $z_{0} \gg L_{D}$ corresponds to the Förster transfer being just a weak perturbation with respect to the stationary solution of the diffusion equation in an isolated organic layer, so the simple boundary condition $\rho(0)=0$ is incorrect. In the case of weak transfer, it is easy to find $z_{0}$ by perturbation theory, starting from the zero-approximation solution of Eq. (A4) with $\Gamma(z)=0$, which is just $\rho^{(0)}(z)=1$. Substituting it in the right-hand side of Eq. (A4) and integrating once over $z$, one obtains $\rho^{(1)}(z)$ with a finite slope at $z \rightarrow \infty$ :

$$
\frac{1}{z_{0}}=\left.\frac{\partial \rho}{\partial z}\right|_{z \rightarrow \infty}=\int_{0}^{\infty} \frac{\tau \Gamma(z)}{L_{D}^{2}} d z,
$$

which gives Eq. (5) for the rate $\Gamma(z)$ given by Eq. (3b).

Focusing on Eq. (A4) with $\Gamma(z)$ given by Eq. (3b), one may notice that the equation contains only one dimensionless parameter, $R_{0}^{3} /\left(L_{D}^{2} a\right)$. As discussed earlier, we expect it to be of the order of unity in the most optimistic case, and smaller if the Förster transfer is inefficient for some reason (large dielectric mismatch, thick barrier, or weak absorption in the quantum well). Eq. (5) is valid when the transfer is weak, that is $R_{0}^{3} /\left(L_{D}^{2} a\right) \ll 1$. Now we make the crucial observation: when $R_{0}^{3} /\left(L_{D}^{2} a\right) \sim 1$, i. e., when Eq. (5) ceases to be valid, we already have $z_{0} \sim a \ll L_{D}$, so Eq. (4) is not sensitive to the precise value of $z_{0}$, and the boundary codition $\rho(0)=0$ gives the correct result. Only when $z_{0} \sim L_{D}$ (or larger), the simple boundary condition $\rho(0)=0$ is wrong and the actual value of $z_{0}$ is important. Thus, Eqs. (4), (5), in fact, correctly give the transfer efficiency for all reasonable values of parameters.

For a scrupulous reader, we note that, strictly speaking, for the rate $\Gamma(z)$ given by Eq. (3b), the boundary condition (A2) is always incorrect, and the large- $z$ asymptotics (A5), in fact, never holds. The reasons for this are discussed in detail in the rest of this Appendix, the bottom line being that Eqs. (4), (5) turn out to give the practically correct result nevertheless. As discussed above, in the limit $R_{0}^{3} /\left(L_{D}^{2} a\right) \ll 1$ the perturbative treatment of Eq. (A4) with $\Gamma(z)$ given by Eq. (3b), starting from the zero-order solution $\rho^{(0)}(z)=1$, gives the firstorder correction

$$
\rho^{(1)}(z)=\frac{R_{0}^{3}}{L_{D}^{2} a} \frac{z^{2}}{2 a(z+a)}=\frac{R_{0}^{3}}{L_{D}^{2} a}\left[\frac{z}{2 a}+\frac{1}{2}+O(a / z)\right] .
$$

The first term in the square brackets provides the leading contribution to the slope, the second term gives a correction to the constant, and the rest does not contribute to the large- $z$ asymptotics. Let us proceed and calculate the second-order correction:

$$
\begin{aligned}
\rho^{(2)}(z)= & \frac{1}{2}\left(\frac{R_{0}^{3}}{L_{D}^{2} a}\right)^{2} \times \\
& \times\left[\frac{z}{3 a}-\ln \frac{z+a}{a e^{5 / 6}}-\frac{a}{z+a}+\frac{a^{2} / 6}{(z+a)^{2}}\right]= \\
= & \frac{1}{2}\left(\frac{R_{0}^{3}}{L_{D}^{2} a}\right)^{2}\left[\frac{z}{3 a}+\frac{5}{6}-\ln \frac{z}{a}+O(a / z)\right] .
\end{aligned}
$$

The last expression, besides the first and the second terms which represent corrections to the slope and to the constant, respectively, contains also a logarithmic term. Because of the latter, the asymptotic form of Eq. (A5) does not hold, and the derivation of the boundary condition, described in Sec. III, is, strictly speaking, invalid. Physically, the transfer rate $\Gamma(z) \propto 1 / z^{3}$ turns out to be too long-range, so that short-distance and long-distance effects cannot be properly separated.

Expression (3b) gives a good approximation when the majority of the excitations in the semiconductor quantum well is created sufficiently close to the organicinorganic interface at $z=0$. As will be seen later, this is true in the most important case of $W \gg a$. The effect of the finite quantum well width can be studied using the expression

$$
\Gamma(z)=\frac{1}{\tau}\left[\frac{R_{0}^{3}}{(z+a)^{3}}-\frac{R_{0}^{3}}{(z+W)^{3}}\right],
$$


obtained by the integration of $1 / r^{6}$ over the slab $-W<$ $z<-a$. Such a simple expression is valid only if one neglects the dielectric constant mismatch between the organic layer, the semiconductor quantum well, and the substrate, but contains the relevant physics to discuss the validity of the effective boundary conditions.

The finite width of the quantum well modifies the asymptotics of $\Gamma(z)$ at $z \gg W$, which becomes $\Gamma(z) \propto$ $1 / z^{4}$ [see Eq. (A11)]. Thus, $W$ provides the upper cutoff for the logarithm and also determines the length scale at which the effective boundary condition, Eq. (A2), can be used (namely, at $z \gg W$ ). However, the problem is back when the quantum well is too thick, $W \gg \min \left\{L, L_{D}\right\}$, since then there is no window $W \ll z \ll L, L_{D}$ where one could match the two asymptotic forms. In this case, the logarithm should be cut off at $z \sim \min \left\{L, L_{D}\right\}$, and $z_{0}$ is defined only with logarithmic precision [i. e., neglecting $O(1)$ in comparison with $\left.\ln \left(\min \left\{L, L_{D}\right\} / a\right)\right]$.

In practice, however, the presence of the logarithm does not spoil too much the validity of Eqs. (4), (5) for the transfer efficiency. Indeed, when $R_{0}^{3} /\left(L_{D}^{2} a\right) \ll 1$, the logarithm is suppressed by a small factor. Even when $R_{0}^{3} /\left(L_{D}^{2} a\right) \sim 1$, the precise value of $z_{0}$ affects Eq. (4) only when $z_{0} \gtrsim L_{D}$, so the logarithm can spoil the final result only when $\min \left\{L, L_{D}, W\right\} \gtrsim a \exp \left(L_{D} / a\right)$, which does not seem to be realistic. As a consequence, for the cases of practical interest, Eq. (3b) can be used rather than Eq. (A11) and, typically, the most efficient transfer rate can be estimated using Eq. (4) and Eq. (5) (or even setting $\left.z_{0}=0\right)$.

There is another formal problem, arising when the main mechanism of exciton diffusion in the organic layer is the Förster transfer betwen organic molecules. Then, strictly speaking, the second spatial derivative in the diffusion equation should be replaced by

$$
\frac{\partial^{2} \rho}{\partial z^{2}} \rightarrow a^{\prime} \int_{0}^{\infty} d z^{\prime} \theta\left(\left|z-z^{\prime}\right|-a^{\prime}\right) \frac{\rho\left(z^{\prime}\right)-\rho(z)}{\left(z-z^{\prime}\right)^{4}}
$$

where $a^{\prime}$ is a short-range cutoff of the order of the lattice constant in the organics (which, generally speaking, does not have to exactly coincide with $a$, responsible for the transfer between the organics and the semiconductor), and $\theta$ denotes the step function which excludes the region $\left|z-z^{\prime}\right|<a^{\prime}$ from the integral. Normally, one expands $\rho\left(z^{\prime}\right)$ around $z^{\prime}=z$ to the second order in $z^{\prime}-z$, and taking advantage of the convergence of the integral, replaces the integral operator by the differential one. However, a detailed inspection of Eq. (A4), modified by replacement (A12), shows that it does not admit an asymptotic solution in the form $\rho(z \rightarrow \infty)=A+B z$, and thus is not equivalent to the differential equation. Still, in the perturbative regime, $R_{0}^{3} \ll L_{D}^{2} a$, we expect the estimate (5) to be valid, as it ensures the overall particle conservation, and the perturbative regime is the only one when the actual value of $z_{0}$ matters, as discussed above. Also, we expect the long-range nature of the transport across the organics only to improve our estimates for the efficiency, so a detailed study of effects of the replacement (A12) is beyond the scope of this paper.
1 V. M. Agranovich, Yu. N. Gartstein, and M. Litinskaya, Chem. Rev. 111, 5179 (2011).

2 V. M. Agranovich, D. M. Basko, G. C. La Rocca and F. Bassani, J. Phys. Cond. Matt. 10, 9369 (1998).

${ }^{3} \mathrm{H}$. Haug and S. W. Koch, Quantum theory of the optical and electronic properties of semiconductors, 5th ed. (World Scientific, Singapore, 2009).

4 §. Schmitt-Rink, D. S. Chemla and D. A. B. Miller, Adv. Phys. 38, 89 (1989).

5 V. M. Agranovich, D. M. Basko, G. C. La Rocca and F. Bassani, Synth. Met. 116, 349 (2001).

6 N. Peyghambarian, H. M. Gibbs, J. L. Jewell, A. Antonetti, A. Migus, D. Hulin, and A. Mysyrowicz, Phys. Rev. Lett. 53, 2433 (1984).

7 D. S. Chemla and D. A. B. Miller, JOSA B 2, 1155 (1985).

8 A. Honold, L. Schultheis, J. Kuhl and C. W. Tu, Phys. Rev. B 40, 6442 (1989).

9 L. Kappei, J. Szczytko, F. Morier-Genoud and B. Deveaud, Phys. Rev. Lett. 94, 147403 (2005).

10 M. Pope and C. E. Swenberg, Electronic Processes in Organic Crystals and Polymers, (Oxford University Press, Oxford, 1999).

11 O. Simpson, Proc. Roy. Soc. (London) A238, 402 (1957).

12 M. R. Philpott, J. Chem. Phys. 59, 4406 (1973).
13 M.I. Alonso and M. Garriga, Thin Solid Films 455-456, 124 (2004).

14 M. D. Galanin, Luminescence of Moleculs and Cristals (Cambridge International Science Publication, Cambridge, 1996).

15 M. L. Cohen and J. R. Chelikowsky, Electronic structure and optical properties of semiconductors, (Springer-Verlag, Berlin 1989).

16 Handbook of optical constants of solids, edited by E. D. Palik (Academic Press, Orlando, 1985).

17 D. M. Basko, G. C. La Rocca, F. Bassani, and V. M. Agranovich, Eur. Phys. J. B 8, 353 (1999).

18 S. Kawka and G. C. La Rocca, Phys. Rev. B 85, 115305 (2012).

19 S. Forrest, Chem. Rev. 97, 1793 (1997).

${ }^{20}$ G. Witte and C. Wöll, J. Mat. Res. 19, 1889 (2004).

21 R. R. Lunt et al., J. Appl. Phys. 105, 053711 (2009).

22 M. S. Bradley and V. Bulovic, Phys. Rev. B 82, 033305 (2010)

23 C. Weisbuch, M. Nishioka, A. Ishikawa and Y. Arakawa, Phys. Rev. Lett. 69, 3314 (1992)

24 I. Carusotto and C. Ciuti, "Quantum fluids of light" (Rev. Mod. Phys., in the press; arXiv:1205.6500v1). 